\title{
Las redes sociales verticales en los sistemas formales de formación inicial de docentes
}

\section{Vertical social networks in formal teachers training systems}

\author{
Melchor GÓMEZ GARCÍA, Rodrigo FERRER y Agustín DE LA HERRÁN \\ Universidad Autónoma de Madrid
}

Recibido: Julio 2014

Aceptado: Febrero 2015

\begin{abstract}
Resumen
Presentamos un estudio sobre redes sociales en la universidad y las posibilidades de mejora que ofrecen en la formación inicial de los futuros maestros.

Se ha integrado una red social en el programa de estudio de Grado de Magisterio de la Universidad Autónoma de Madrid y se ha registrado el uso que hacen de ella los estudiantes y el impacto que estas tecnologías tienen en su actividad académica y su socialización. El formato elegido es el de red vertical o red propia, que ofrece posibilidades de gestión y seguridad adaptables a los requerimientos de los docentes.

Se ha llevado a cabo un estudio de caso en una red social vertical propia con tres grupos de estudiantes de Grado de Magisterio (futuros docentes) siguiendo un proceso de recogida sistemática de datos.

Como comprobaremos, su uso está muy extendido como elemento de comunicación social y ofrece mejoras en su aplicación académica desde el punto de vista de la interacción educativa y la motivación formativa. Pero la integración que tiene en su formación actual es pequeña en relación a las posibilidades y aplicaciones que tiene en otros campos.
\end{abstract}

Palabas clave: tecnología educativa, redes sociales, formación inicial del profesorado, enseñanza superior, estrategias de aprendizaje.

\begin{abstract}
A study of social networks in university is presented in this paper in which the potential for improvement in the future teacher initial training was studied.

Integrated social networking was integrated in the Bachelor of Education of the Universidad Autonoma Madrid as well as a recording of the students using it. The impact that the use of these technologies has on their academic activities and socialization was studied. We have chosen vertical network format or home network, which offers management and security capabilities and is adaptable to teachers requirements.

We conducted a case study in its own vertical social network with three groups of students from Teachers Training (future teachers) following a process of systematic data collection.
\end{abstract}


As it was shown by the study, their use is widespread as an element of social communication and offers improvements in academic use, like educational interaction or motivation. But integration in its current formation is small compared to the possibilities and applications that might be found in other fields.

Keywords: technology uses in education, social networks, teacher education, higher education, learning strategies.

Internet impregna cada recodo de nuestra actividad diaria y tiene una evolución frenética y difícilmente predecible. Estos rápidos pasos en su evolución los intenta seguir y asimilar la sociedad en tiempo record. Uno de los elementos actuales que está teniendo mayor impacto lo constituyen las Redes Sociales, que en este vertiginoso entorno nos plantean cuestiones diversas, referidas a su poder de comunicación, a su inmediatez, a su integración social o a sus beneficios. Pero ¿estamos preparados para su uso? ¿Nuestros jóvenes conocen los posibles peligros que estas redes pueden entrañar? ¿Podemos formarnos a través de ellas?

La universidad atraviesa actualmente una etapa de integración de plataformas digitales de enseñanza en la formación reglada (Sánchez Rodríguez, 2009). Pero estos sistemas de gestión del aprendizaje o Learning Management System (LMS), tienen un uso docente que habitualmente replica modelos tradicionales de enseñanza y aprendizaje (Valjataga, Pata, \&Tammets, 2011) y no aprovecha el potencial de comunicación y colaboración que puede proporcionar Internet para formarnos.

La red ofrece herramientas en línea que tienen enormes posibilidades para adaptarse a las particularidades de los estudiantes y paralelamente facilitar su proceso digital de enseñanza-aprendizaje (Dixon, 2012). Estas herramientas en línea universalizan el uso de determinados recursos por su fácil acceso, su sencillez de manejo y su flexibilidad y adaptación al perfil personal.

Estas conllevan también ventajas y plantean dudas, pues permiten la "creación de entornos más flexibles para el aprendizaje, la eliminación de las barreras espaciotemporales entre docente y alumno, un incremento de las modalidades comunicativas, favorecer escenarios y entornos de carácter interactivo, el autoaprendizaje y el trabajo en grupo, salir de los muros del recinto educativo, ofrecer nuevas formas de tutoría y orientación del alumnado y favorecer la formación de carácter permanente” (Cabero, 2003: 105).

Las redes sociales (digitales), como herramientas en línea que son, constituyen entornos Web a los que se accede con un perfil personal y en los que podemos compartir documentos (fotos, textos, vídeos...) e interactuar con otros usuarios a través de nuestro perfil, de manera que se van creando grupos de personas por cercanía de intereses (Gómez y Solís, 2010).

Los jóvenes utilizan redes sociales de modo asiduo en diferentes ámbitos de su vida -ocio, amistad, compras- y por tanto si se utilizan también en entornos académicos permiten un modo formación más acorde con las maneras de actuar que los estudiantes tienen en sus otros entornos de su vida. "Si queremos dar una enseñanza acorde con los tiempos que vivimos los medios han de formar parte de ello" (Marín, 2009: 102). Las 
redes sociales se perfilan así como potentes recursos para el aprendizaje y se constituyen en flexibles herramientas de comunicación educativa. El recurso se personaliza en cada estudiante abriendo así las posibilidades formativas para cada alumno de manera específica.

\section{Los jóvenes españoles y las redes sociales}

Es una evidencia que los jóvenes españoles usan las redes sociales. Según el IAB Spain Research de 2013, casi el 80\% de los internautas españoles mayores de 18 años usa redes sociales, con un incremento continuado durante los últimos años. Y si las redes sociales son un fenómeno social, lo son especialmente y de manera más acusada entre los jóvenes, que son el sector con mayor tasa de utilización.

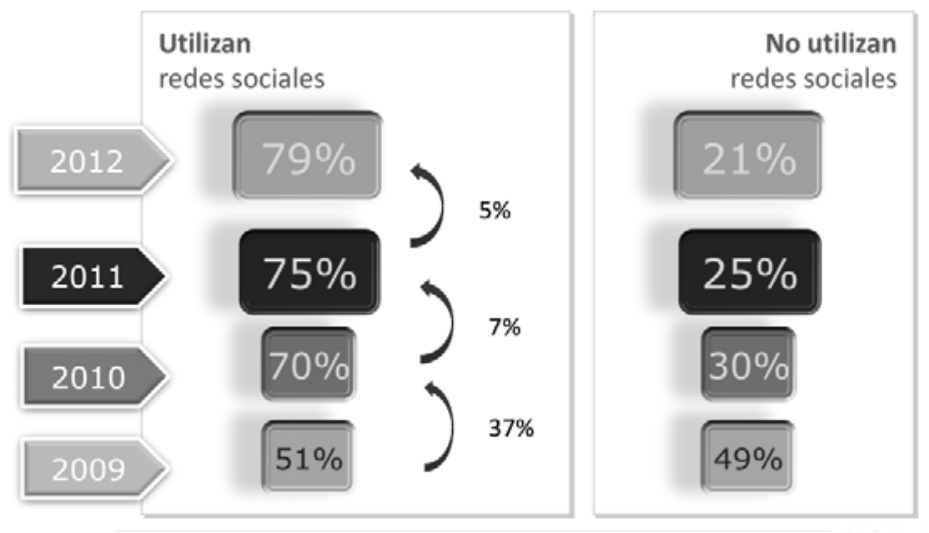

Figura 1. ¿Cuáles de los servicios de internet utilizan los jóvenes? (IAB Spain Research)

Este crecimiento continuado sigue, pero en menor medida que en años anteriores, lo que nos indica que estamos entrando en la fase de madurez, y por tanto de incluir este recurso como un elemento más en nuestra actividad vital y en el aprendizaje en particular.

Además no sólo crece el sector de población que las usa, sino que también aumenta el tiempo que se les dedica. El aumento de redes existentes y el acceso móvil convierten a las redes en una cotidianidad. Según el estudio anterior en 2012 casi el $80 \%$ de los internautas las utilizaban a diario.

El móvil está siendo sin duda el gran compañero de viaje de esta tendencia: el acceso a las redes se ha disparado en estos dispositivos y está facilitando su expansión y su uso casi omnipresente. Desde 2012 el acceso desde este tipo de dispositivos es mayor que el hasta ahora habitual acceso desde el ordenador. Dado que el acceso al móvil se tiene cada vez con más temprana edad y que los estudiantes y profesores disponen de estos elementos tecnológicos, las razones para su integración educativa 
son mayores y más consistentes. La posibilidad de contar en estos dispositivos con muchas herramientas Web, entre las que están las redes sociales, posibilita experiencias nuevas dentro y fuera del aula (Ruiz \& Sánchez, 2010).

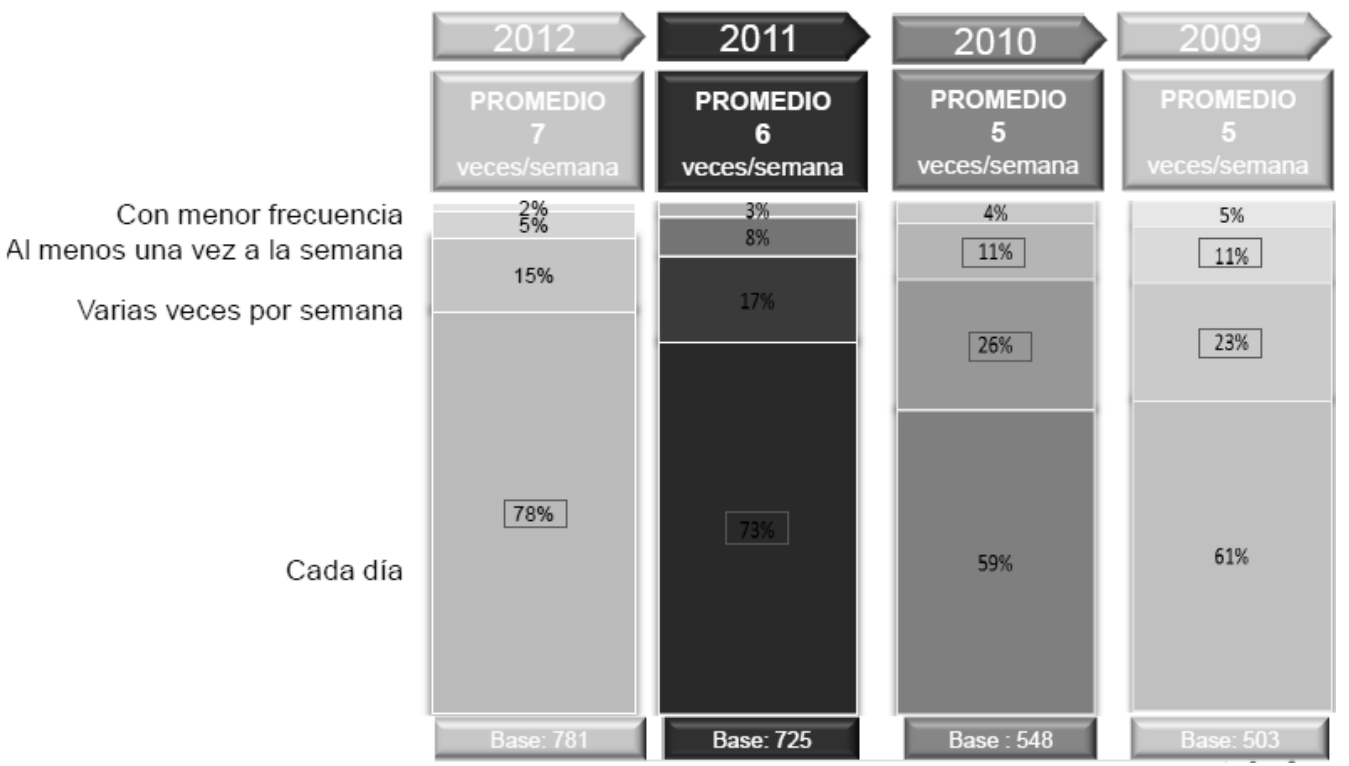

Figura 2. ¿Con qué frecuencia consultas las redes sociales? (IAB Spain Research)

Partiendo de la idea de que no se puede crear una realidad distinta en los centros educativos a la que los estudiantes tienen en sus casas, llegamos a al planteamiento siguiente: si ellos se conectan a Internet y usan las redes sociales, habrá que enseñarles a hacer un uso responsable de ellas e incluso ir más allá y beneficiarnos de su uso como una herramienta formativa más. Por tanto, una tarea apasionante consistirá en orientar la actividad educativa de modo que los estudiantes aprendan a usarlas de forma adecuada, y a aprovecharlas para aprender. Habrá, por tanto, que formarles en el uso, pero no sólo a ellos, sino a los profesores, al entorno del estudiante y especialmente a los futuros profesores.

\section{Las redes sociales en educación}

Cuantos más cauces existan para comunicarnos en una comunidad (como la comunidad en la que se aprende), más información relevante estará al alcance de los participantes, y por tanto, mejor será la experiencia dentro de ella. El gran valor de las redes sociales aplicadas a la educación radica en el contacto social y en el hecho de utilizarlas como recursos de comunicación, pues la educación se realiza a través de la comunicación. 
Una red social en educación es un ambiente creado en Internet al que el estudiante puede acceder desde el aula, desde su casa, o desde cualquier otro sitio en que esté, que le permite tener muchas más formas de acceso al aprendizaje que desde el aula y muchas más horas que las horas de clase. Además, en dicho ambiente, el estudiante puede aprender sobre el tema que le interese, donde alguien pondrá una semilla y habrá otros estudiantes y docentes que le ayuden y complementen generando una sinergia que fomenta el aprendizaje.

Las posibilidades que ofrecen las redes sociales como modelo multidireccional de comunicación generan multitud de perspectivas. En una dirección son un elemento para favorecer el papel activo del alumnado. En otra dirección suponen una conexión educativa potente que acerca al profesor, al utiliza el "mismo lenguaje" que los alumnos. Son además canales de comunicación entre centros educativos, sociedad y familia, que fortalecen por tanto la interacción entre las instituciones educativas y el resto de agentes sociales. En definitiva, facilitan una propuesta más abierta, un aula como espacio flexible e interactivo para el aprendizaje y sus aplicaciones futuras más allá del centro educativo.

Las redes sociales ofrecen claras oportunidades al sector universitario y aunque los estudiantes de grado viven conectados y muestran una actitud positiva en utilizar las redes sociales con fines educativos, siguen sacando poco provecho académico de ellas. (Gómez-Aguilar, Roses-Campos y Farias-Batlle, 2012). En la mayoría de los casos, el uso de las redes para actividades académicas casi siempre parte de la iniciativa de los alumnos y casi nunca por iniciativa del profesor, lo que lleva a que los estudiantes ni siquiera sigan a sus profesores en las redes populares.

Las mismas tendencias positivas en la actitud de los alumnos se reflejan en el estudio de Espuny, González, Lleixà y otros (2011). En él los estudiantes no sólo mostraron una actitud positiva respecto al uso didáctico de las redes sociales, sino que mayoritariamente afirmaba que utilizar las redes sociales en tareas de aprendizaje no les supone un esfuerzo adicional, sino que al contrario descubren en ellas múltiples ventajas a la hora de compartir información, realizar trabajos, interactuar entre ellos y con los profesores.

Pero el escaso aprovechamiento en la educación formal de las posibilidades de comunicación y de colaboración que permiten las redes sociales (Richmond, Rochefort y Hitch, 2011) arranca probablemente en el arraigo en las universidades de la enseñanza tradicional formal, donde la comunicación es unidireccional (profesoralumno) y donde al alumno es poco proclive a participar.

Es conveniente un aprovechamiento académico de las redes sociales, dada la profundidad de su implantación en las rutinas diarias de los estudiantes universitarios. La actitud positiva del alumnado y las posibilidades comunicativas posibilitan su utilización didáctica. Si bien el reto está en despertar el interés de instituciones, docentes y alumnado para integrarlas como herramientas básicas de la enseñanza (Castañeda, 2010).

Pero no todas las redes son iguales, ni tienen las mismas funcionalidades y no todas tienen las mismas ventajas o inconvenientes. Conocerlas, estudiarlas con sentido crítico 
y aprender a utilizar con los estudiantes las que mejor se adapten a su formación es una ventaja y una mejora en nuestro quehacer como profesores (Whals, 2010).

"Los servicios de redes sociales más apropiados para la educación son aquellos que permiten la creación de redes independientes de otras y que permiten el aislamiento del resto de los usuarios de Internet, mediante la creación de espacios seguros.” (Haro, 2010:7) Estas redes son conocidas como redes sociales privadas de carácter vertical, o "redes verticales".

El uso de redes sociales en educación puede, por tanto, aportarnos diversos beneficios en el trabajo con nuestros alumnos (Haro, 2008) si somos capaces de canalizar correctamente su uso:

- Permiten centralizar en un único sitio todas las actividades docentes de un grupo que aprende.

- Aumentan el sentimiento de comunidad educativa entre los alumnos y entre alumnos y profesores debido al efecto de cercanía que producen.

- Mejoran el ambiente de trabajo ya que permiten al alumno crear objetos cercanos a su interés, así como los propios del trabajo que requiere la educación.

- Hacen más sencilla y fluida la comunicación entre profesores y alumnos.

- Facilitan la coordinación entre diversos grupos de aprendizaje (clase, asignatura, grupo de alumnos de una asignatura, etc.) mejorando el trabajo realizado.

- Integran el comportamiento social básico por parte de los alumnos: qué puedo decir, qué puedo hacer, hasta dónde puedo llegar, etc.

Esta función social que facilitan las redes digitales constituye además una meta que los estudiantes persiguen con frecuencia. Existe una amplia evidencia de que los estudiantes que persiguen determinadas metas sociales en la escuela triunfan académicamente (Wentzel, 2001). Incluso se podrían explicar ciertas variaciones en el rendimiento académico por los distintos grupos de metas sociales que persiguen los estudiantes, lo que nos lleva a considerar a la motivación como otro campo a tener en cuenta.

La motivación es considerada por la psicología como un estado o un proceso interno que activa, orienta y mantiene la conducta (Acosta, 1998). Desde el aprendizaje social, la motivación es considerada como la base fundamental para el aprendizaje en cualquier nivel de la educación y reconoce en ella un concepto vital en cualquier teoría pedagógica (Bandura, 1986), reconociendo que la motivación es un fenómeno complejo que está condicionado por un gran número de factores que deben ser contemplados en el proceso educativo por el docente.

Algunas teorías sobre la motivación en el aprendizaje se plantean independientes del contexto en el cual se desarrolla el proceso formativo (presencial, apoyado con TIC, virtual), pero otras plantean una estrecha relación. Esta relación entre contexto y 
motivación es analizado en algunos casos en medios digitales, como en la teoría del apoyo proactivo (Simpson 2008), que identifica diversas variables que permiten propiciar la motivación en un entorno digital. En este entorno debemos observar el contacto proactivo, el acercamiento temprano del tutor al estudiante, con el planteamiento de que el éxito en el aprendizaje radica en centrarse en las fortalezas personales y el aprovechamiento de los talentos de los estudiantes.

La mayoría de los individuos suelen comprometerse en las tareas que requieren dedicación y esfuerzo por dos tipos de razones: aprender y rendir. Se implican en la tarea bien por su deseo de aprender y mejorar sus capacidades o bien para demostrar sus capacidades y buscar las valoraciones positivas de los demás. En el campo de la educación estas tipologías se concretan en estudiantes que sostienen metas de aprendizaje para incrementar su capacidad y aquellos que optan por metas de rendimiento para demostrar su capacidad (Elliot, 1999).

Estos deseos de los estudiantes de ser valorados socialmente en la clase, incluyendo el éxito académico, podrían ser parte de un sistema motivacional más global derivado de experiencias de socialización. Esta perspectiva asume que la adopción y persecución de metas socialmente apropiadas en un determinado contexto, nace de una necesidad más general de formar vínculos interpersonales y experimentar una sensación de pertenencia y relación con la sociedad (Dweck, 2001).

Las redes sociales tienen infinidad de usos y de perfiles. Desde la publicación de información en sus diferentes formatos, a la elaboración de micromensajes, pasando por los espacios para compartir vídeos o la geolocalización de datos. Pero el uso de estas redes implica un cambio en la organización y en la metodología (Boyd, 2009).

Las redes sociales son el nuevo componente de la sociedad digital, que está generando un cambio total en el poder de los medios de comunicación tradicionales. Pero mientras que radio y televisión son gobernadas por la dirección del propio medio, que decide qué transmitir y qué no, en las redes sociales el poder de elección es igualitario y todos podemos comunicar nuestro pensamiento a gran parte de la sociedad. Pero las redes sociales públicas están plagadas de publicidad disfrazada de opiniones, y si no tenemos un conocimiento adecuado de estos recursos corremos el riesgo de ser manipulados.

Las redes sociales son la réplica en Internet del mundo real pero con elementos que potencian sus posibilidades, como el posible anonimato o la rapidez e inmediatez de lo que allí surge. En el mundo real los deben convivir con ciertos peligros (gente desconocida, engaños, malas intenciones) y para prevenirles se desarrollan diferentes estrategias que van desde acompañarlos por la calle, a mostrarles los peligros o instruirles en la defensa frente a los mismos. Replicar esta educación en las redes sociales no es tan sencillo, y es ahí donde surge la necesidad de disponer de una red social privada y propia de la institución educativa, que facilite educar y formar a los estudiantes en un entorno que es atractivo para ellos y similar a su entorno de interacción habitual, pero a la vez está protegido de actividades externas.

Por ello, la creación de una red vertical propia, independiente de las redes sociales comerciales, nos permite aislar a nuestros estudiantes del resto de los usuarios de 
Internet (o al menos de aquellos que no tienen autorización), prevenirles de contenidos inapropiados, eliminar la contaminación publicitaria y protegerles de acciones que nada tienen que ver con la formación. Con este tipo de red, las características informales de las redes sociales, el dinamismo, la inmediatez, y toda su atracción se aprovechan para la formación, minimizando los riesgos y educando sobre ellos. También se consigue así capitalizar la adicción (o parte de ella) que las Redes Sociales provocan en una red donde se intercambia el material de estudio, las dudas, los temas de interés, etc.

En la Universidad Autónoma de Madrid se ha apostado por este modelo vertical y hemos creado nuestra propia red, a la que hemos llamado SocialUAM y a la que se accede desde la Web de la universidad o directamente desde la dirección http://social.uam.es. Esta red está basada en la plataforma Elgg, por ser esta una plataforma de servicios de red social de código abierto y por tanto habernos permitido modificar la apariencia y adaptarla a nuestros requerimientos. Esta nos ofrece microblog, trabajo en red, comunidad, recolección de noticias vía feeds e intercambio de archivos. Todo puede ser compartido entre los usuarios, utilizando los controles de acceso y puede ser catalogado mediante etiquetas. Elgg además de ser libre y de código abierto tiene una alta calidad técnica y gran reconocimiento, con premios internacionales como mejor plataforma social de trabajo en red de código abierto (InfoWorld 2009 de International Data Group).

En SocialUAM los alumnos pueden crear sus propios contenidos de acuerdo a sus intereses y pueden ser acompañados y guiados por otros estudiantes o por profesores que se interesen en el tema. A la vez SocialUAM es accesible desde cualquier lugar que disponga de conexión a Internet, por lo que la dedicación en horas puede ser mayor y su caracterización como red social la hacen atractiva para los estudiantes.

Las redes sociales empiezan a tener una innegable utilidad para la educación formal y además permiten establecer un vínculo con lo informal. Esta unión produce una retroalimentación que favorece el proceso educativo general. En este sentido, SocialUAM permite la creación de espacios formales dentro de la red para el intercambio de materiales, novedades y tareas correspondientes a una asignatura, como lo puede constituir un grupo (similar a los grupos de Facebook) donde los alumnos realizarán las tareas, descargarán material propuesto por el docente, eventualmente podrán complementarlo compartiendo otros documentos, realizar consultas y aportes.

Los alumnos también pueden solicitar la creación de grupos sobre sus intereses, intercambiar sus ideas y aportar a las ideas de otros, generando un aprendizaje alternativo, informal y sobretodo motivador, lo cual logra que cada alumno se desempeñe y profundice en lo que más le interesa y apasiona. Las redes sociales encauzan así las dos funciones deseables en las TIC: por un lado, "facilitar la obtención de materiales educativos” y, por otro, "entretener y motivar” (Cabero, 2000).

Esta red, como todas las redes verticales, está accesible desde Internet por lo que una parte de la misma está pública y visible para el público en general, pero solo pueden aportar contenidos los usuarios acreditados de la UAM, esto es, solo los estudiantes matriculados en la Universidad Autónoma de Madrid pueden participar y 
solo ellos pueden acceder a la totalidad de los apartados, aunque haya otra parte pública y visible para todos los internautas.

El paso a utilizar una red social vertical frente a los sistemas digitales anteriores (plataforma Web, blogs o wikis) se ha dado tras reflexionar algunos aspectos que se consideraban de interés:

- No era posible en algunos de ellos restringir el acceso al material, con el consiguiente mal uso o uso desautorizado del material.

- No era posible la interacción entre los alumnos, generar discusiones, aportes, etc.

- No era posible por parte de los estudiantes crear contenidos nuevos, hacer propuestas, crear grupos, y otras acciones propias de las necesidades de los alumnos (que son justamente el mayor atractivo de las redes sociales)

\section{Metodología}

El objetivo de este estudio que ha sido financiado parcialmente gracias al proyecto S2013-ICE2715 (eMadrid) ha sido profundizar en el conocimiento del uso educativo de las redes sociales a través de la evaluación de la incidencia de diferentes aspectos relacionados con el aprendizaje y la comunicación. En este resumen valoraremos el impacto únicamente de tres parámetros: la integración como recurso de aprendizaje, la acción socializadora y el elemento motivador.

1. Respecto a la integración como recurso de aprendizaje, hemos querido conocer datos sobre el uso previo a la actividad en aula, el uso durante el horario escolar y el uso posterior a la actividad del aula.

2. En cuanto a la acción socializadora hemos investigado sobre la incidencia en el diagrama de relaciones entre los alumnos y el acceso a los círculos de trabajo en grupo, los círculos de aula y círculos diferentes a los del grupo de trabajo.

3. Para el elemento motivador hemos medido situaciones que nos diesen información sobre el índice para el uso indicado en las tareas de clase y el uso libre.

En nuestra investigación hemos elegido el estudio de caso en una red social vertical propia para tres grupos de estudiantes de Grado de Magisterio (futuros docentes) de la Universidad Autónoma de Madrid, siguiendo un proceso de recogida sistemática de datos. A través de este método de investigación se puede conocer profundamente una realidad educativa para comprenderla y así tratar de mejorarla (Álvarez \& San Fabián, 2012). Para ello hemos elegido una muestra pequeña pero significativa, y hemos profundizado con técnicas de recogida de información variadas que nos facilitan el registro digital de estos sistemas de red y que garantizan un rigor. La potencialidad investigadora de un estudio de caso se apoya en el supuesto de que lo global se refleja en lo local. 
En el curso en el que se realizó la investigación participaron en la red tres grupos de estudiantes de grado de 57, 63 y 60 alumnos, de primero y segundo curso, que utilizaron la red social vertical propia de la universidad como cauce de gestión del trabajo durante un cuatrimestre en tres de las asignaturas de TIC y Sociedad Digital que se impartieron en dicho cuatrimestre. La coordinación del estudio se llevó a cabo por los tres profesores de los respectivos grupos de clase y se contó con la colaboración de tres estudiantes de doctorado vinculados al tema.

La experiencia de la red social digital para grupos de estudiantes del caso objeto de estudio se inició en el curso 2011-2012, con la realización de diversas experiencias puntuales del uso de la red social (actividades de un día) en grupos piloto, para limar aspectos funcionales de seguridad y acceso. Desde ese mismo curso surgió un creciente interés por parte de otros estudiantes por utilizar las redes sociales como herramienta formativa desde un punto de vista formal, y las solicitudes de formación por parte de otros profesores en el uso de esta herramienta como recurso docente. Así, durante el curso 2012-2013 se realizaron experiencias continuadas que cubrían el periodo completo de impartición de asignaturas, e integradas en la programación docente. Desde el grupo de proponentes de la red social estábamos convencidos de sus posibilidades educativas pero también estábamos seguros de que muchos aspectos eran mejorables, por lo que nos propusimos hacer una revisión de la experiencia de modo sistemático para optimizar el funcionamiento y la utilización de la red, así como para recoger las opiniones de los estudiantes y nuestras reflexiones tras la acción.

Hemos utilizado el registro de actividad de usuarios que nos proporciona Elgg para rastrear tanto la transcripción directa de los diálogos que se han mantenido como los datos cuantitativos de actividad de los estudiantes (número de conexiones, número de participaciones, tiempo de conexión...). Esta fuente directa de información se ha estado acompañada con diversos sistemas Web de análisis de datos para compartir la información e interpretarla en el equipo de investigación.

El estudio ha estado organizado en tres fases:

1. Adaptación de las actividades formativas.

Las guías didácticas y las programaciones de las asignaturas se han adaptado para realizarse con el apoyo de la red social, como elemento comunicador y de transmisión de la información. Para ello se han tenido que redefinir algunas actividades y tareas que habitualmente se realizaban con medios no digitales, o con medios informáticos de uso local. Los profesores adaptaron sus propuestas de trabajo con los alumnos para integrar el proceso dentro de una actividad en red: enunciando las tareas a través de twits, proponiendo trabajo en parejas en comunicación por la red, solicitando la entrega de tareas a través de Internet, proponiendo actividades de redacción y mejora del lenguaje en el microblog y elaborando listas y grupos de la red para la mejora de la sociabilidad. Con estas indicaciones los alumnos tenían la opción de trabajar con la red como elemento vehicular, pero con el fondo epistemológico de las asignaturas que se integraron en la propuesta. 
2. Diseño y aplicación de un cuestionario de recogida de información.

Antes de la puesta en práctica de la tarea se elaboraron diversos cuestionarios para recoger la información. Un grupo de cinco especialistas (dos en Métodos de Investigación y Diagnóstico en Educación, y tres en Didáctica y Organización Escolar) se encargó de su validación.

Se ha utilizado un cuestionario electrónico cerrado estructurado siempre al comienzo del curso y antes de la puesta en práctica de la experiencia. Una vez ha ido avanzada la experiencia y ya casi en su fase final se he pasado un segundo cuestionario. En los casos en que se ha podido se ha pasado un tercer cuestionario con posterioridad a la experiencia.

A la información aportada por los cuestionarios se ha añadido la aportada por los registros de actividad que la propia red social ofrece, relativo al número de intervenciones (notas escritas), el número de amigos (personas que leen), los mensajes directos (similar al correo electrónico) y las listas subscritas. Estos datos tienen la ventaja añadida de ser datos directos y objetivos, sin sesgo ni matiz. Los cuestionarios se han realizado online y han sido anónimos en cuanto a la respuesta emitida, pero controlando el acceso a las personas que respondían al mismo.

\section{Formación y puesta en práctica.}

Durante el transcurso del cuatrimestre se ha llevado a cabo esta experiencia, en la que cada alumno disponía de un ordenador personal en clase. Las actividades se han organizado en módulos de actividad que comenzaban siempre con sesiones presenciales en el aula de clase, en las que se explicaban las tareas a realizar y se indicaban los materiales de apoyo y las herramientas de las que se iban a disponer (entre las que se encontraba de modo vehicular la red social). Posteriormente se abría un periodo de trabajo, por parte de los alumnos, de un modo autónomo, tanto en grupo como individualmente, en el que han contado como elemento de apoyo y comunicación la red Social UAM.

En mitad de este periodo se convocaba una sesión de resolución presencial de dudas, de carácter voluntario y a la que solo asistían los estudiantes que requerían de más ayuda que la proporcionada en la red social. Aunque la entrega de trabajos se ha hecho siempre de modo digital, finalmente se llevaba a cabo una sesión de aula en la que los alumnos comentaban en grupos con los docentes las vicisitudes acaecidas durante la realización del trabajo, y tras la entrega del mismo. Todas estas reuniones se han grabado y transcrito para su posterior análisis, generando documentos escritos que han servido de apoyo al análisis del equipo de investigación. 


\section{Resultados}

Los datos se han obtenido de las encuestas realizadas a los estudiantes y del registro de actividad que genera Elgg. De los datos obtenidos expondremos a continuación únicamente los referidos a los tres aspectos planteados en este artículo.

\section{Uso y aprendizaje}

Un dato inicial nos refleja que cuantitativamente el número de estudiantes que usa redes sociales es similar al del resto de jóvenes de su mismo tramo de edad, en torno al $80 \%$. Pero si miramos más detenidamente vemos que las expectativas que genera la posibilidad de utilizarlas para su aprendizaje son ya superiores a la utilización media en esas edades. Incluso cabe un dato más optimista, por el hecho de que el registro de uso que finalmente se ha hecho de la red es superior a las propias expectativas que se tenían inicialmente.

Aunque las actividades se han diseñado teniendo en cuenta la posibilidad de usar la red social, siempre se ha contado con la opción de realizar dichas tareas a través de otras herramientas digitales distintas. A pesar de ello, el uso de Social UAM ha sido generalizado, llegando casi al 95\% de los participantes. Este es un dato destacable por lo alto que ha sido, y más teniendo en cuenta las expectativas que desde diferentes lugares nos presagiaban cuando propusimos el uso de la red y la supuesta dificultad para integrar éste tipo de herramientas en una dinámica formativa.

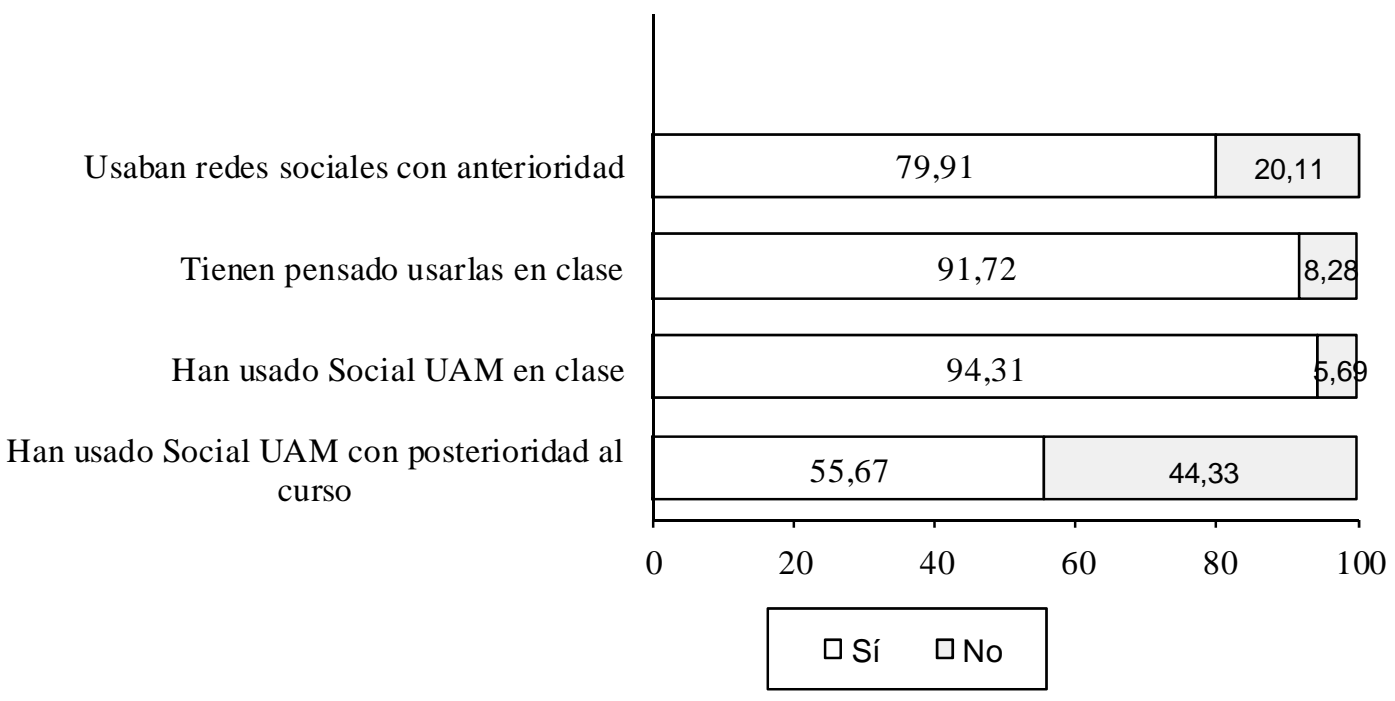

Gráfico 1. Uso de la red social (porcentaje)

El uso de la red en periodos posteriores a la experiencia registra un importante descenso, bajando a casi la mitad, por razones que podremos analizar posteriormente. 


\section{Socialización}

El registro de actividad de los alumnos en lo referente a los seguidores y los usuarios seguidos ("amigos"), los grupos y las relaciones en general nos ofrece resultados que permiten reflexionar si la interacción de los alumnos se ha limitado al grupo de trabajo o se han abierto nuevos círculos de relación. También nos permite vislumbrar si esta interacción se ha restringido a las actividades propias de la tarea encomendada o se han podido enriquecer con otro tipo de interacciones que superaban las propias de la tarea.

Podemos ver un elemento esperanzador en el uso de redes verticales al observar que dos de cada tres alumnos logró sobrepasar su círculo de compañeros de trabajo propuesto. Es decir, tenemos un número significativo de alumnos que entablaron relación con personas de otras clases (11,12\%), al que hay que unir el grupo de los que entablaron relación con personas de su clase pero no solo de su grupo de trabajo (56,77\%). Este elemento es importante desde el punto de vista del auge cada vez mayor que tiene el desarrollo de habilidades sociales y de trabajo en colaboración en el perfil profesional, que reclaman las empresas a nuestros estudiantes.

Por el contrario, el número de alumnos que solo establecieron relación con las personas con las que tuvieron que trabajar por requerimiento de la actividad también fue importante (más del 20\%).

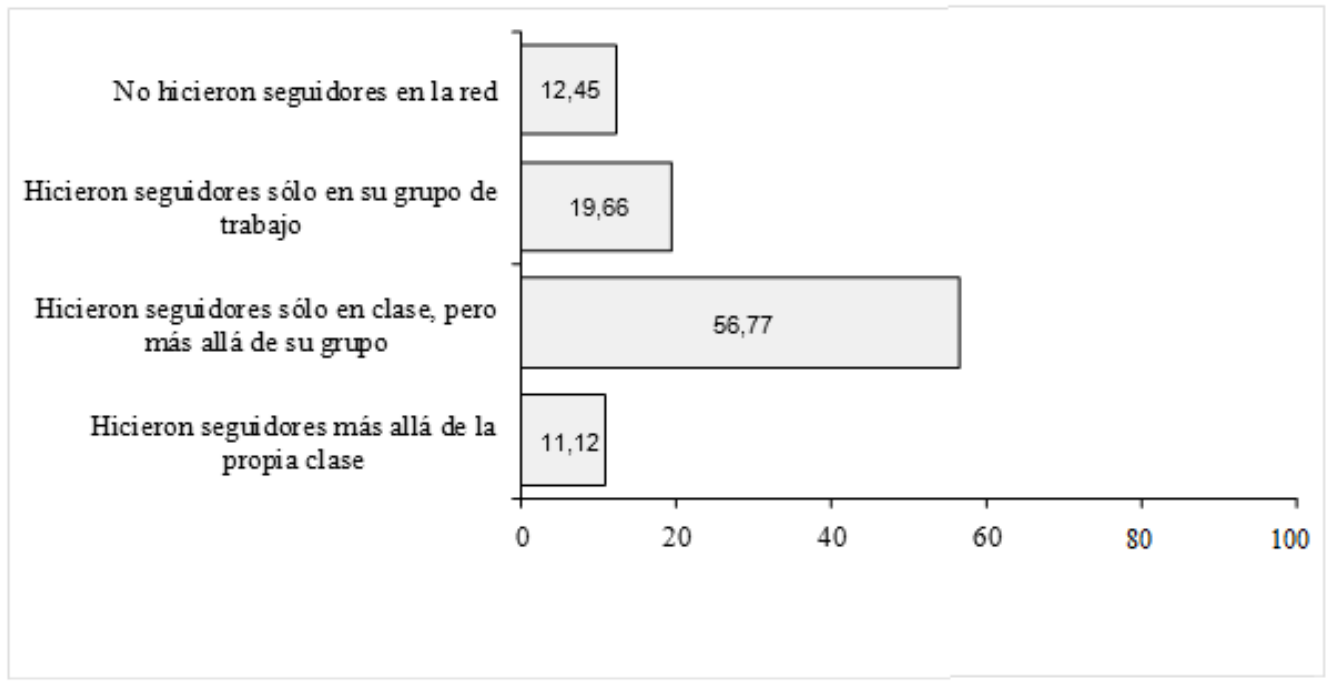

Gráfico 2. Uso de aplicaciones de grupos B (Social UAM) 


\section{Datos sobre la motivación}

Para estudiar la naturaleza de mensajes enviados hemos elaborado una clasificación de los mensajes (los conocidos como twits o mensajes cortos de no más de 124 caracteres) en diferentes categorías, y atendiendo a diversos criterios cuantitativos y cualitativos. En este apartado presentamos solo dos de estos criterios: la referencia (o no) a temas académicos y haber sido requeridos (o no) por la actividad a realizar. Los mensajes recogidos que atienden a estos dos elementos los vemos recogidos en la siguiente tabla.

\begin{tabular}{|c|c|c|c|}
\hline \multicolumn{2}{|c|}{ Los mensajes enviados son... } & Enviados en \% & Enviados en \% \\
\hline \multicolumn{2}{|c|}{ Menos mensajes de los requeridos } & $9,11 \%$ & $9,11 \%$ \\
\hline \multicolumn{2}{|c|}{ Sólo los mensajes requeridos } & $38,35 \%$ & $38,35 \%$ \\
\hline \multirow{2}{*}{$\begin{array}{l}\text { Mas mensajes de } \\
\text { los requeridos por } \\
\text { la clase }\end{array}$} & $\begin{array}{l}\text { Con información de la } \\
\text { clase }\end{array}$ & \multirow{2}{*}{$52,54 \%$} & $25,73 \%$ \\
\hline & $\begin{array}{l}\text { Con información externa } \\
\text { a la clase }\end{array}$ & & $26,81 \%$ \\
\hline
\end{tabular}

Tabla 1. Naturaleza y cantidad de mensajes enviados.

Vemos de entrada dos datos cuantitativamente positivos. Primero el mayoritario porcentaje de estudiantes que han mandado más twits de los requeridos por la propia actividad, y segundo el minoritario número de alumnos que no cubren los requerimientos mínimos de la actividad. El primer dato es interesante porque los alumnos que supuestamente han realizado más actividad de la solicitada (que podríamos decir alumnos sobremotivados) no solo es el mayor porcentaje, sino que es más de la mitad. El segundo dato es interesante por el hecho de que la proporción de estudiantes que no alcanzan los requisitos establecidos no sólo es el menor, sino que no llega siquiera a uno de cada diez alumnos.

De los subapartados obtenidos entre los estudiantes con más actividad de la requerida, tenemos dos grupos casi iguales de alumnos que circunscriben esta actividad exclusivamente a asuntos académicos y de alumnos que utilizan la red como elemento de comunicación social, más allá de lo puramente académico.

\section{Conclusiones}

Las redes sociales verticales tienen un alto grado de aceptación en la formación superior. Esto se observa desde el planteamiento de la herramienta como recurso docente y las expectativas de uso de la red vertical en educación formal por parte de los estudiantes, que han sido mayores de las que tienen estos jóvenes para otros usos no académicos. Y estas expectativas se han concretado posteriormente en un uso real mayor que ha alcanzado casi el 95\% de usuarios (gráfico 1).

El uso de este tipo específico de redes es eminentemente académico, como podemos ver en la tabla I, en la que observamos que apenas 1 de cada 4 mensajes es ajeno a la 
actividad puramente formativa (26,81\%). Esta afirmación justifica que una vez terminado el periodo lectivo, la tasa de uso de la red descienda a casi la mitad. Este uso específicamente académico se refleja también en el gráfico 2, al observar que apenas uno de cada diez usuarios interaccionó en modo grupal (haciendo amigos) con usuarios de aulas diferentes a la suya, y por tanto con actuaciones supuestamente diferentes a las de la tarea encomendada. Con ello nos podemos reafirmar en lo acertado de la elección de la red de tipo vertical, pues cumple su principal propósito, que es la comunicación de carácter formativo.

Dado que la mayoría de los mensajes que se registraron eran de carácter académico y que más del $66 \%$ de los estudiantes se relacionaron con otros alumnos diferentes a los de su grupo de trabajo (dato que sale de la suma de los usuarios que hicieron seguidores más allá de su grupo de trabajo y los usuarios que hicieron seguidores más allá de su clase, gráfico 2), podemos deducir que los estudiantes se comunican con los usuarios de otros grupos para temas formativos en la mayoría de las ocasiones. Las redes sociales digitales facilitan entonces la comunicación con otros compañeros para aprender. No podemos decir que para aprender mejor, pero sí que con ellas se comunican mucho.

La casi totalidad de los participantes ha manejado la red social del modo solicitado por el docente, cumpliendo con los requisitos propuestos. Si en la tabla I unimos los usuarios que enviaron las tareas requeridas (38,35\%) y los que enviaron más de las requeridas (52,54\%) tenemos que más del $90 \%$ ha cumplido con los requisitos propuestos en la actividad con la red social, y por tanto ha hecho un uso adecuado de la misma. Este dato apoya de un modo evidente la supuesta sencillez de esta herramienta y su apariencia amigable, que intuimos desde el punto de vista de la casi inexistente instrucción en su manejo, y el uso autónomo que han tenido los usuarios. La simple consideración de ese 52,54\% de usuarios que tuvieron más actividad en la red de la propuesta para la tarea, permite considerar estas herramientas sociales en red como un facilitador de la interacción educativa, pues como ya hemos comentado, estas comunicaciones son mayoritariamente de tipo académico.

Si además observamos que el $9,11 \%$ de estudiantes que no han realizado las actividades mínimas requeridas en la red social pueden haber realizado las tareas encomendadas a través de otros recursos de los que disponían (como la plataforma digital), llegamos a una valoración aún más positiva en lo referente a la buena acogida de la propuesta por parte de los estudiantes.

Un aspecto menos optimista surge en la observación de que casi la mitad de los alumnos aplicó estrictamente los mensajes solicitados por la actividad o incluso menos, lo que podría interpretarse como una motivación exclusivamente académica en la realización de la actividad. Los estudiantes han cumplido con lo planteado, pero no han ido más allá.

Las redes sociales verticales no cubren la función de comunicación social que cubren las redes sociales horizontales (Facebook, Twitter, Linkedin...). A pesar de que en la tabla I podemos observar un grupo de cerca del $25 \%$ de usuarios que escribe mensajes no académicos, éste porcentaje es bajo observado en forma global. De hecho, 
ese $25 \%$ de mensajes no académicos se efectúan a través de esta red probablemente por la comodidad de hacerlo en el mismo entorno en el que están desarrollando su actividad de aprendizaje. Pero aun así, la mayoría de ellos es evidente que sigue utilizando sus canales habituales en otro tipo de redes sociales. Otro dato que corrobora esta conclusión es el hecho de que una vez acabado el periodo lectivo el uso de la red desciende a menos de la mitad (gráfico 1).

Las redes sociales verticales pueden ser un elemento comunicativo importante dentro de la propia institución universitaria (aunque no lo sea externamente), pues entre los estudiantes que hicieron las actividades con la red social y tuvieron más actividad de la requerida para las tareas, hubo mucha comunicación con alumnos de otros cursos y promociones, lo que ha impulsado el conocimiento entre estudiantes de diferentes años. Es decir, podemos considerar que estas herramientas ayudan a avivar las interacciones sociales entre los alumnos y a romper los tradicionales grupos que producen cierto aislamiento. Este aspecto no podría desarrollarse en otro tipo de red que no fuera vertical y propio.

Sería muy interesante poder indagar en estudios posteriores cuánto, cómo y qué mejora este tipo de relación digital frente a la tradicional y escasa relación presencial entre alumnos de distintos años y promociones. Con el paso del tiempo puede ser especialmente interesante poder estudiar este tipo de comunicación entre los alumnos egresados y los propios estudiantes del año.

\section{Referencias bibliográficas}

ACOSTA, M. (1998). Motivación y rendimiento académico en los estudios superiores. Málaga: Ediciones Aljibe.

ÁLVAREZ, C. y SAN FABIÁN, J. L. (2012). La elección del estudio de caso en la investigación cualitativa. Gazeta de Antropología, vol 28, pp. 1-12. Recuperado de http://www.ugr.es/ pwlac/G28_14Carmen_Alvarez-JoseLuis_SanFabian.pdf, julio 2012.

BANDURA, A. (1986). Social foundations of thought and action: A social cognitive theory. Englewood Cliffs: Prentice Hall.

BOYD, D. M. (2009). Living and Learning with Social Media. Penn State Symposium for Teaching and Learning with Technology. State College, PA. Recuperado de http://www.danah.org/papers/talks/PennState2009.html, octubre 2013

CABERO, J. (2000). Las nuevas tecnologías y las transformaciones de las instituciones educativas. En Lorenzo, M. y colaboradores (Coords.), Las organizaciones educativas en la sociedad neoliberal (pp. 463-493). Granada: Grupo Editorial Universitario.

CABERO, J. (2003). La galaxia digital y la educación: los nuevos entornos de aprendizaje. En J. I. Aguaded (Dir.). Luces en el laberinto audiovisual (pp. 102121). Huelva: Comunicar, Grupo Ágora Digital y Universidad de Huelva. 
CASTAÑEDA, L. (2010). Aprendizaje con redes sociales. Tejidos educativos para los nuevos entornos. Sevilla: MAD.

DIXON, B. (2012). Social Media for School Leaders: A Comprehensive Guide to Getting the Most Out of Facebook, Twitter, and Other Essential Web Tools. New York: Jossey-Bass

DWECK, C.S. (2001). Motivación social: metas y procesos sociocognitivos. En J. Juvonen y K.R. Wentzel (Eds.), Motivación y adaptación escolar (pp. 217-234). México: Oxford.

ELLIOT, A.J. (1999). Approach and avoidance motivation and achievement goals. Educational Psychologist, 34, 169-189.

ESPUNY, C., GONZÁLEZ, J., LLEIXÁ, M. y GISBERT, M. (2011). Actitudes y expectativas del uso educativo de las redes sociales en los alumnos. Revista de Universidad y Sociedad del Conocimiento (RUSC), 8(1), pp. 171-185.

GÓMEZ, M. y SOLÍS, J. I. (2010). Ser padres en un mundo digital. Madrid: Anaya Multimedia.

GÓMEZ-AGUILAR, M.; ROSES-CAMPOS, S. Y FARIAS-BATLLE, P. (2012). El uso académico de las redes sociales en universitarios. Comunicar: Revista Científica de Comunicación y Educación, 19 (38), pp. 131-138.

DE HARO, J. J. (2008). Las redes sociales en educación. Recuperado de http://jjdeharo.blogspot.com.es/2008/11/la-redes-sociales-en-educacin.html, octubre 2013

DE HARO, J. J. (2010). Redes Sociales en Educación. Recuperado de http://www.chaval.es/chavales/sites/default/files/editor/05cap-redes-sociales-parala-educacion.pdf, octubre 2013

IAB SPAIN RESEARCH (2013). IV Estudio Anual Redes Sociales. Recuperado de http://www.iabspain.net/wp-content/uploads/downloads/2013/01/IV-estudio-anualRRSS_reducida.pdf, octubre 2013

MARÍN, V. (2009). La formación docente universitaria a través de las TIC. Pixel-Bit. Revista de Medios y Educación, vol 35, pp. 97-103. Recuperado de http://www.sav.us.es/pixelbit/pixelbit/articulos/n35/8.pdf, octubre 2013

RICHMOND, N.; ROCHEFORT, B. Y HITCH, L.P. (2011). Using Social Networking Sites During the Career Management Process. En Wankel, L.A. y Wankel, C. (Eds.), Higher Education Administration with Social Media: Including Applications in Student Affairs, Enrolment Management, Alumni Affairs, and Career Centers (pp. 147-164). Bingley (UK): Emerald.

RUIZ, J. Y SÁNCHEZ, J. (2010). Investigaciones sobre buenas prácticas con TIC. Málaga: Ediciones Aljibe.

SÁNCHEZ, J. (2009). Plataformas de enseñanza virtual para entornos educativos. Pixel-Bit. Revista de Medios y Educación, vol 34, pp. 217-233 
SIMPSON, O. (2008). Motivating learners in open and distance learning: do we need a new theory of learner support? Open Learning: The Journal of Open and Distance Learning, 23(3), pp. 159-170.

VALJATAGA, T.; PATA, K. Y TAMMETS, K. (2011). Considering students' perspective on personal and distributed learning environments. En Lee, M. y McLoughlin, C. (Eds.), Web 2.0-based E-Learning: Applying social informatics for tertiary teaching (pp. 85-107). Hershey, PA: IGI Global. Recuperado de http://unifi.academia.edu/mariachiarapettenati/Papers/944467/Personal_knowledge _management_skills_in_web_2.0-based_learning, octubre 2013

WENTZEL, K.R. (2001). Metas sociales y relaciones sociales como motivadoras de la adaptación a la escuela: un análisis motivacional. En J. Juvonen y K.R. Wentzel (eds.), Motivación y adaptación escolar (pp. 269-294). México: Oxford.

WHALS, K. (2010). 100 ways to teach with Twitter. New York : EmergingEdTech. Recuperado de http://www.emergingedtech.com/2010/02/100-ways-to-teach-withtwitter/, octubre 2013

\section{Correspondencia con autores}

Melchor GÓMEZ GARCÍA

Universidad Autónoma de Madrid

C/Hierbabuena 20. 28770. Colmenar Viejo (Madrid)

e-mail: melchor.gomez@uam.es

Agustín DE LA HERRÁN

Facultad de Formación de Profesorado y Educación.

Universidad Autónoma de Madrid

Campus de Cantoblanco, 28049 Madrid

e-mail: agustin.delaherran@uam.es

Rodrigo FERRER

Escuela de Magisterio ESCUNI

Avda. Niza, portal 7-E, 4 D. 28022 Madrid

e-mail: rferrer@escuni.com 